\title{
Tidal heating in Enceladus
}

\author{
Jennifer Meyer*, Jack Wisdom \\ Massachusetts Institute of Technology, Cambridge, MA 02139, USA
}

Received 20 February 2007; revised 3 March 2007

Available online 19 March 2007

\begin{abstract}
The heating in Enceladus in an equilibrium resonant configuration with other saturnian satellites can be estimated independently of the physical properties of

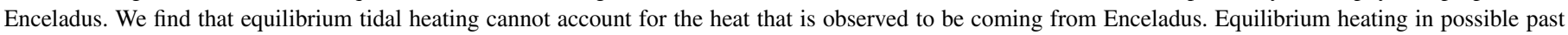
resonances likewise cannot explain prior resurfacing events.
\end{abstract}

(c) 2007 Elsevier Inc. All rights reserved.

Keywords: Enceladus; Saturn, satellites; Satellites, dynamics; Resonances, orbital; Tides, solid body

\section{Introduction}

Enceladus is a puzzle. Cassini observed active plumes emanating from Enceladus (Porco et al., 2006). The plumes consist almost entirely of water vapor, with entrained water ice particles of typical size $1 \mu \mathrm{m}$. Models of the plumes suggest the existence of liquid water as close as $7 \mathrm{~m}$ to the surface (Porco et al., 2006). An alternate model has the water originate in a clathrate reservoir (Kieffer et al., 2006). Both models require substantial energy input to drive the plumes. The plumes originate in the features dubbed the "tiger stripes," in the south polar terrain. The heat emanating from the south polar terrain has been estimated to be $5.8 \pm 1.9 \mathrm{GW}$ (Spencer et al., 2006). So some heating mechanism provides about $6 \mathrm{GW}$ of energy to the system. The estimated rate of radiogenic heating is $0.32 \mathrm{GW}$, and the estimated current rate of tidal heating resulting from the small orbital eccentricity of Enceladus is about $0.12 \mathrm{GW}$, for an assumed $k_{2}$ of 0.0018 and a $Q$ of 20 (Porco et al., 2006). So these sources of heating are inadequate.

Squyres et al. (1983) remark that even if the current rate of tidal heating was sufficient to maintain Enceladus in an active state, much greater heating would be required to initiate the process. They suggest that heating of order $25 \mathrm{GW}$ is necessary to initiate melting, and propose that this might have been obtained by a much larger orbital eccentricity.

Any mechanism for supplying the required energy must pass the "Mimas test" (Squyres et al., 1983). Mimas has an ancient surface, but is closer to Saturn than Enceladus and has a larger orbital eccentricity. Any mechanism that is proposed to heat Enceladus must not substantially heat Mimas. Using the conventional tidal heating formula (Peale and Cassen, 1978; Peale, 2003), the estimated tidal heating in Mimas is about 11 times the heating in Enceladus, if the rigidity of the two bodies is the same. Thus conventional tidal heating in the current orbital configuration does not pass the test.

\footnotetext{
* Corresponding author.

E-mail addresses: meyerj@mit.edu (J. Meyer),wisdom@mit.edu (J. Wisdom).
}

One mechanism for heating Enceladus that passes the Mimas test is the secondary spin-orbit libration model (Wisdom, 2004). Fits to the shape of Enceladus from Voyager images indicated that the frequency of small amplitude oscillations about the Saturn-pointing orientation of Enceladus was about 1/3 of the orbital frequency. In the phase-space of the spin-orbit problem near the damped synchronous state the stable equilibrium bifurcates into a period-tripled state. If Enceladus were trapped in this bifurcated state, then there could be several orders of magnitude greater heating than that given by the conventional tidal heating formula. What was special about Enceladus compared to Mimas was its shape. New fits of the shape to Cassini images of Enceladus showed that Enceladus was not near the 3:1 secondary resonance, but, remarkably, was near the 4:1 secondary resonance (Porco et al., 2006). A similar analysis shows that if caught in this secondary resonance, the system could again be subject to several orders of magnitude additional heating. Unfortunately, the predicted libration was not seen. An upper limit placed on the magnitude of the libration was 1.5 degrees, which in turn places an upper limit on the heating from the secondary resonance mechanism of $0.18 \mathrm{GW}$ (Porco et al., 2006). So if the limits of the libration amplitude are reliable, then the secondary resonance spin-orbit mechanism is ruled out for the present system. It may still be possible that the system was locked in this resonance in the past. Note that the large heating that would result from libration in the secondary resonance would damp the orbital eccentricity, and at sufficiently small eccentricity the secondary resonance becomes unstable. Thus the secondary resonance mechanism could at most produce an episode of heating.

Lissauer et al. (1984) suggested that Enceladus might have recently been involved in a 2:1 mean motion resonance with Janus. Janus is evolving outwards due to torques from Saturn's rings. At present, Janus is just $1000 \mathrm{~km}$ outside the resonance. Only a few tens of millions of years ago Janus was at the resonance. If Janus encountered the resonance when the eccentricity of Enceladus was low, the probability of capture into the $e$-Enceladus resonance would be high. They found that if Janus and Enceladus were trapped in the resonance and were in an equilibrium configuration then Enceladus would be subject to $4.5 \mathrm{GW}$ of heating, which is comparable to the observed heating. But the model has numerous limitations (Peale, 2003). The value of the mass of Janus has been revised 
downwards, and this leads to smaller tidal heating (see below). The angular momentum in the A-ring is limited, so the resonance could only have persisted for a limited time in the past. More importantly, Enceladus shows evidence of multiple resurfacing episodes. The resonance with Janus could at most explain the most recent activity. In addition, the model has to appeal to an impact to get the system out of the resonance. The alternative escape mechanism suggested is that the Janus resonance became unstable when the Enceladus-Dione resonance was reached. But this seems unlikely, as Enceladus and Dione are not deeply in the resonance and Dione has little effect on the orbital evolution of Enceladus at present (Sinclair, 1983). There may also be a problem damping down the implied equilibrium eccentricity of Enceladus to the present low value of 0.0047 in the short time (tens of millions of years) since the resonance was purportedly disrupted. Actually, the simplest scenario for the encounter of Janus with the 2:1e-Enceladus Janus-Enceladus resonance is that Janus just passed through the resonance with little effect on the orbit of Enceladus. In this scenario Janus encounters Enceladus at its current eccentricity, but at this eccentricity the system has a low probability of being captured by the resonance. We find that the capture probability at the current eccentricity of Enceladus is only $0.7 \%$.

There are other possibilities for resonance configurations involving Enceladus in the past (see Fig. 1). Perhaps tidal heating in these resonances was responsible for past resurfacing events. These resonances include the 3:2 MimasEnceladus and the 3:4 Enceladus-Tethys resonances. If the $Q$ of Saturn is sufficiently low numerous other resonances could have been encountered. Evolution through these has not been studied in detail, but we can estimate the equilibrium tidal heating expected while trapped in the resonances (see below).

Ross and Schubert (1989) investigated tidal heating in Enceladus using multilayered viscoelastic models of the satellite. They find that equilibrium heating in a homogeneous Maxwell model at the current eccentricity can be as large as $920 \mathrm{GW}$. The heating is proportional to the Love number of the satellite and in the viscoelastic models the dynamic Love number can be orders of magnitude larger than the elastic Love number. They also investigate heating in a two layer model consisting of a conductive elastic lithosphere overlying a Maxwell interior and a three layer model with a liquid waterammonia layer between the lithosphere and the Maxwell core. These models are tuned to give a heating rate of about $4 \mathrm{GW}$, similar to that found by Lissauer

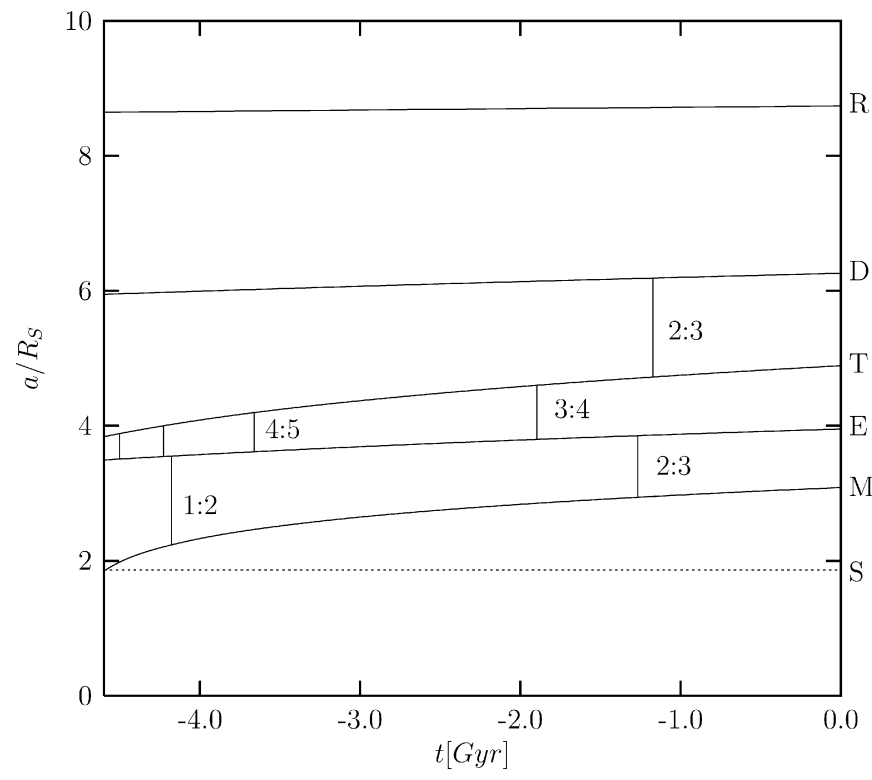

Fig. 1. The approximate locations of the first-order resonances among the saturnian satellites are shown for $Q_{S}=18,000$. The shift of position of the resonances due to Saturn's oblateness has been ignored. Also shown are the tidally evolved orbits as a function of time. The dotted line shows the synchronous radius. The minimum $Q_{S}$ is determined by placing Mimas at the synchronous radius at the beginning of the Solar System. The current 2:1 and 4:2 resonances between Enceladus-Dione and Mimas-Tethys are not shown. et al. (1984) in their Janus model. These models require a low conductivity insulating layer. Thus, it appears to be possible for tidal heating to provide enough input energy to account for the observed energy output from Enceladus. The Mimas test is not addressed by these models; it seems likely that if similar viscoelastic models were applied to Mimas then there would also be large tidal heating in Mimas, contradicting its cold inactive state. Nevertheless, viscoelastic enhancement of the Love number has been presented as a simple solution to the problem of heating Enceladus (Spencer et al., 2006; Stevenson, 2006).

In this paper we calculate the equilibrium rates of tidal heating in Enceladus independent of the physical properties of Enceladus, based on conservation of energy and angular momentum. We find that tidal heating in Enceladus is much less than the observed radiated heat.

\section{Heating from torques}

One mechanism of heating is tidal dissipation in a synchronously rotating satellite. As a system evolves deeper into an eccentricity-type resonance, the eccentricity grows. As the eccentricity of a satellite grows the rate of energy dissipation in the satellite grows, with the square of the orbital eccentricity. Dissipation of energy in a satellite tends to damp the eccentricity. As tidal torques push the system deeper into resonance, the eccentricity grows, until the rate of growth is balanced by the rate of decay, due to the internal dissipation. At equilibrium, the eccentricity no longer changes and there is a steady state rate at which angular momentum is transferred to the outer satellite. The rate of angular momentum transfer is related to the rate of heating in the satellites. The equilibrium rate of heating can be calculated using conservation of energy and angular momentum (Lissauer et al., 1984).

The angular momentum $L$ and energy $E$ of a satellite of mass $m$ in an Keplerian orbit of semimajor axis $a$ about a primary of mass $M$ are

$L=m \sqrt{G M a\left(1-e^{2}\right)}$,

$E=-G M m /(2 a)$.

The rate of change of the Keplerian energy can be related to the applied torque. The energy can be written in terms of the angular momentum and eccentricity: $E=\tilde{E}(L, e)$. Let $n=\sqrt{G M / a^{3}}$; we have

$\frac{\partial \tilde{E}}{\partial L}=\frac{n}{\sqrt{1-e^{2}}} \approx n$,

ignoring corrections of order $e^{2}$. The rate of change in angular momentum is the torque

$\frac{\mathrm{d} L}{\mathrm{~d} t}=T$.

Ignoring the change in energy due to the change in eccentricity, the rate of change in orbital energy from an applied satellite torque is

$\frac{\mathrm{d} E}{\mathrm{~d} t}=\frac{\partial \tilde{E}}{\partial L} \frac{\mathrm{d} L}{\mathrm{~d} t}=\frac{n T}{\sqrt{1-e^{2}}} \approx n T$,

again ignoring corrections of order $e^{2}$.

Assume there are two satellites, and that there is some resonant interaction between the satellites so that angular momentum can be transferred between them.

For the sake of qualitative reasoning, let us ignore contributions to energy changes due to orbital eccentricities. Consider a small impulsive torque that causes a change in the angular momentum of the system $\Delta L$. For simplicity assume that the torque is applied only to the inner satellite. The energy change due to this angular momentum change is approximately $\Delta E=n_{0} \Delta L$. This is the energy input to the satellite system. Now let us take into account the exchange of angular momentum between the satellites. The change in angular momentum $\Delta L$ is now distributed in some way between the two satellites $\Delta L=\Delta L_{0}+\Delta L_{1}$. The change in energy of the orbits is then $\Delta E=n_{0} \Delta L_{0}+n_{1} \Delta L_{1}$. Because $n_{1}<n_{0}$, this energy change is less than the energy gained by the satellites. The remaining energy goes into heating the satellites. 
The energy input to the system is the sum of the energy inputs for the individual satellites. This presumes there is no cross tidal interaction between the satellites. So the total rate at which energy is transferred to the satellites from the rotation of the planet is $n_{0} T_{0}+n_{1} T_{1}$, ignoring corrections of order $e^{2}$. This energy changes the orbits and heats at least one of the satellites.

So we can write

$n_{0} T_{0}+n_{1} T_{1}=\frac{\mathrm{d}}{\mathrm{d} t}\left(E_{0}+E_{1}\right)+H$,

where $E_{i}$ are the Keplerian energies of the satellites, and $H$ is the rate of heating. We have ignored the gravitational interaction energy of the satellites. If most of the heating is in one satellite, we can take $H$ to be the heating rate of that satellite.

As an eccentricity-type resonance is approached one of the satellite eccentricities grows. Near a $j:(j-1)$ mean motion resonance, the eccentricity depends on a parameter $\delta=j n_{1}+(1-j) n_{0}$ that measures how close the system is to resonance. The condition of resonance equilibrium is that the rate of change of $\delta$ is zero. This implies

$j \frac{\mathrm{d} n_{1}}{\mathrm{~d} t}=(j-1) \frac{\mathrm{d} n_{0}}{\mathrm{~d} t}$.

Close to resonance the parameter $\delta$ is small, so $j n_{1} \approx(j-1) n_{0}$. Dividing these, we find that

$\frac{1}{n_{0}} \frac{\mathrm{d} n_{0}}{\mathrm{~d} t} \approx \frac{1}{n_{1}} \frac{\mathrm{d} n_{1}}{\mathrm{~d} t}$

which in turn implies

$\frac{1}{a_{0}} \frac{\mathrm{d} a_{0}}{\mathrm{~d} t} \approx \frac{1}{a_{1}} \frac{\mathrm{d} a_{1}}{\mathrm{~d} t}$.

Following Lissauer et al. (1984), let us assume that $T_{0} \gg T_{1}$. Conservation of the angular momentum of the system requires

$\frac{\mathrm{d}}{\mathrm{d} t}\left(L_{0}+L_{1}\right)=T_{0}+T_{1} \approx T_{0}$.

Using Eq. (1) at small $e$, we find

$\frac{1}{2} \frac{L_{0}}{a_{0}} \frac{\mathrm{d} a_{0}}{\mathrm{~d} t}+\frac{1}{2} \frac{L_{1}}{a_{1}} \frac{\mathrm{d} a_{1}}{\mathrm{~d} t}=T_{0}$.

Using the equilibrium condition, Eq. (9), we find

$T_{0}=\frac{1}{2 a_{0}} \frac{\mathrm{d} a_{0}}{\mathrm{~d} t}\left(L_{0}+L_{1}\right)$

Using Eq. (6), assuming $n_{0} T_{0} \gg n_{1} T_{1}$, the rate of change in energy of the system is equal to

$n_{0} T_{0}=\frac{\mathrm{d}}{\mathrm{d} t}\left(E_{0}+E_{1}\right)+H$.

Using this equation, Eq. (12), and the equilibrium condition again, we derive

$H=n_{0} T_{0}-\frac{T_{0}}{L_{0}+L_{1}}\left(\frac{G M m_{0}}{a_{0}}+\frac{G M m_{1}}{a_{1}}\right)$.

Again ignoring corrections of order $e^{2}$, we derive

$H=n_{0} T_{0}\left(1-\frac{1+m_{1} a_{0} /\left(m_{0} a_{1}\right)}{1+\left(m_{1} / m_{0}\right) \sqrt{a_{1} / a_{0}}}\right)$.

Lissauer et al. (1984) generalize this formula to three satellites in equilibrium. For the torque on each satellite we use the formula

$T=\frac{3}{2} \frac{G m^{2} R_{S}^{5} k_{2 S}}{a^{6} Q_{S}}$,

where $k_{2 S}$ and $Q_{S}$ are the potential Love number and $Q$ of Saturn, $m$ is the mass of the satellite, $R_{S}$ is the radius of Saturn, and $a$ is the orbit semimajor axis (Schubert et al., 1986). For $k_{2 S}$ we use the value 0.341 (Gavrilov and Zharkov, 1977). The minimum $Q_{S}$ for Saturn may be determined by the condition that Mimas be outside the synchronous orbit at the beginning of the Solar Systemthis gives about $Q_{S} \geqslant 18,000$. A maximum can be placed on $Q_{S}$ if we adopt the tidal origin of the Mimas-Tethys resonance. The age of the Mimas-Tethys resonance for a $Q_{S}$ of 18,000 is $2 \times 10^{8}$ yrs (Sinclair, 1983). Placing the origin of the Mimas-Tethys resonance at the beginning of the Solar System limits the $Q_{S}$ of Saturn to be less than $4 \times 10^{5}$.

The principal resonances that exist now or might have been operative in the recent past that involve Enceladus are: 2:1 Enceladus-Dione, 3:2 MimasEnceladus, 3:4 Enceladus-Tethys, and the 2:1 Janus-Enceladus resonances. For each of these we can calculate the equilibrium tidal heating given the torque on the inner body. This torque is tidal for all but Janus, for which it is a ring torque. Applying the equilibrium heating rate formula to each of these resonances we find, for $Q_{S}=18,000: 2.4 \mathrm{GW}$ for the 2:1 Enceladus-Dione resonance, $0.71 \mathrm{GW}$ for the 3:2 Mimas-Enceladus resonance, $1.2 \mathrm{GW}$ for the 4:3 Enceladus-Tethys resonance, and $0.81 \mathrm{GW}$ for the 2:1 Janus-Enceladus resonance. For the Janus-Enceladus resonance Lissauer et al. (1984) found $4.5 \mathrm{GW}$, but they used the larger mass of Janus determined through Voyager observations. Peale (2003) found $0.95 \mathrm{GW}$ using the pre-Cassini mass of Janus, $2.0 \times 10^{18} \mathrm{~kg}$, determined by Yoder et al. (1989). Keep in mind that Enceladus and Dione may not be in an equilibrium configuration (see below). Also, the assumption that one torque dominates is invalid.

If the torque to the innermost satellite does not dominate then the formula needs to be generalized. Beginning with Eq. (6), we use the resonance condition, Eq. (9), to get

$H=\frac{n_{0} T_{0}}{\sqrt{1-e_{0}^{2}}}+\frac{n_{1} T_{1}}{\sqrt{1-e_{1}^{2}}}-\frac{T_{0}+T_{1}}{L_{0}+L_{1}}\left(\frac{G M m_{0}}{a_{0}}+\frac{G M m_{1}}{a_{1}}\right)$.

The formula readily generalizes to an equilibrium of three satellites, by adding an additional term to each of the sums. And this formula reduces to that of Lissauer et al. (1984) at small eccentricity if $T_{1}$ is set to zero.

Using this formula we recalculate the equilibrium heating rates for each of the resonances given above, assuming $Q_{S}=18,000$. We find: $1.1 \mathrm{GW}$ for the 2:1 Enceladus-Dione resonance, $0.48 \mathrm{GW}$ for the 3:2 Mimas-Enceladus resonance, and $0.75 \mathrm{GW}$ for the 2:1 Janus-Enceladus resonance. The implied heating of the Enceladus-Tethys resonance is negative; this resonance has no equilibrium as the orbits are diverging. Adding the torque on the outer satellite has reduced the heating for all resonances.

For the Enceladus-Dione and Mimas-Enceladus resonances these are upper limits to the heating rates because we have used the lower bound of 18,000 for the $Q$ of Saturn. For larger $Q_{S}$, the torques and heating rates will be proportionally lower (see Fig. 3).

The nonsolar radiated power from Enceladus is estimated to be $5.8 \pm$ 1.9 GW (Spencer et al., 2006). This is larger than all the equilibrium heating rates.

\section{Equilibrium eccentricity}

The equilibrium heating rate corresponds to an equilibrium eccentricity of Enceladus. We can derive the equilibrium eccentricity by requiring that the equilibrium heating rate be equal to the heating rate in a synchronously rotating satellite in an eccentric orbit (Peale and Cassen, 1978; Peale, 2003):

$H=\frac{21}{2} \frac{k_{2 E}}{Q_{E}} \frac{G M_{S}^{2} R_{E}^{5} n}{a^{6}} e^{2}$,

where $k_{2 E}$ and $Q_{E}$ are the potential Love number and $Q$ of Enceladus, and $e$ is the eccentricity. Using Eq. (16) and Eq. (17), we find

$$
\begin{aligned}
e^{2}= & \frac{1}{7 D}\left\{1-\frac{1+m_{1} a_{0} /\left(m_{0} a_{1}\right)}{1+\left(m_{1} / m_{0}\right) \sqrt{a_{1} / a_{0}}}\right. \\
& \left.+\left(\frac{m_{1}}{m_{0}}\right)^{2}\left(\frac{a_{0}}{a_{1}}\right)^{6}\left[\frac{n_{1}}{n_{0}}-\frac{1+m_{1} a_{0} /\left(m_{0} a_{1}\right)}{1+\left(m_{1} / m_{0}\right) \sqrt{a_{1} / a_{0}}}\right]\right\},
\end{aligned}
$$

where $D$ is a measure of the relative strength of tides in Enceladus versus tides in Saturn:

$D=\frac{k_{2 E}}{Q_{E}} \frac{Q_{S}}{k_{2 S}}\left(\frac{M_{S}}{m_{E}}\right)^{2}\left(\frac{R_{E}}{R_{S}}\right)^{5}$.

Thus the equilibrium value of the eccentricity depends on the unknown $k_{2 E} / Q_{E}$ of Enceladus and the unknown $Q_{S}$. Note that if the torque on the 


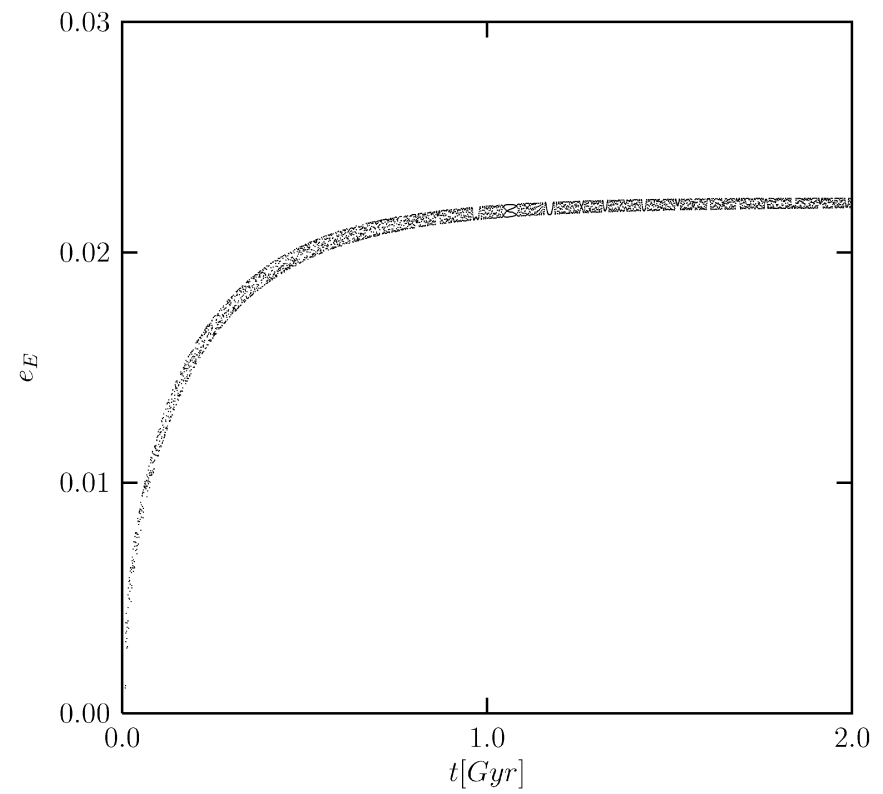

Fig. 2. The eccentricity of Enceladus approaches an equilibrium value as the system evolves into the $e$-Enceladus 3:2 Mimas-Enceladus resonance.

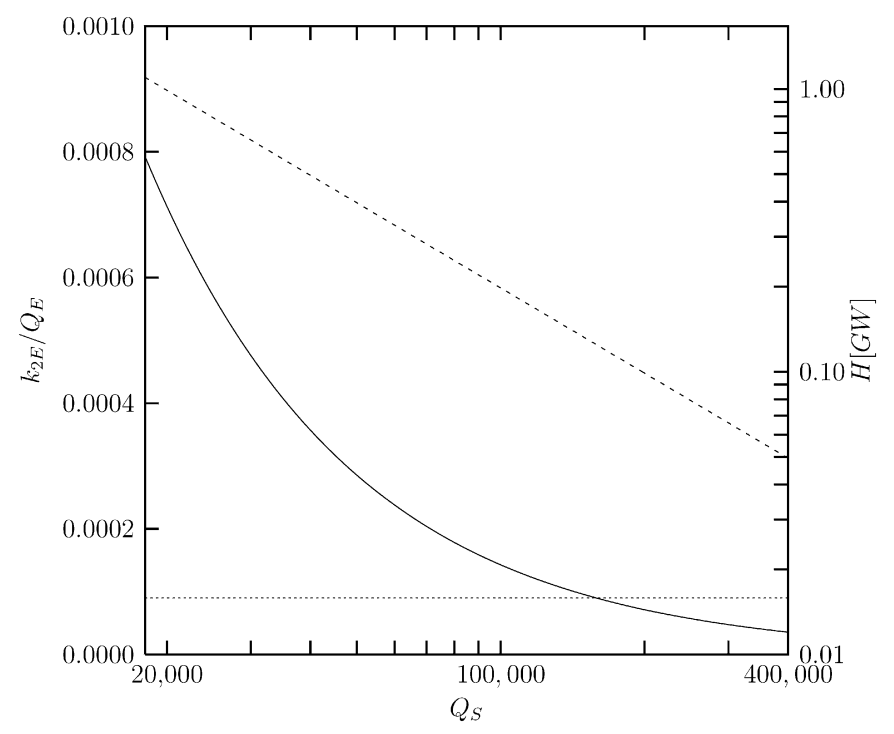

Fig. 3. The solid line shows the $k_{2 E} / Q_{E}$ for which the current configuration of Enceladus (with eccentricity 0.0047) and Dione is a tidal equilibrium for the given value of $Q_{S}$. The dotted line shows the value of $k_{2 E} / Q_{E}$ using Kelvin's formula for the Love number, using a rigidity of $4 \times 10^{9} \mathrm{Nm}^{-2}$, and a $Q$ of 20 . The dashed line gives the equilibrium heating rate $H$ in Enceladus as a function of $Q_{S}$.

outer body is negligible then the term with square brackets in Eq. (19) can be ignored, but for the satellites considered here this is not the case.

We can illustrate the approach to equilibrium and confirm the equilibrium value of the eccentricity by performing numerical integrations of the evolution of the system. Our model is an averaged resonance model that includes terms in the disturbing function up to order $e^{2}$, with dissipative terms that affect both the semimajor axes and eccentricities. We have applied this model to study the evolution into the $e_{E}$-type 3:2 resonance between Mimas and Enceladus. For this resonance, Eq. (19) becomes $e^{2} \approx(59.5 D)^{-1}$. For $k_{2}=0.0018, Q_{E}=$ 100 , and $Q_{S}=18,000$, the equilibrium eccentricity is calculated to be 0.022 . The simulated evolution is shown in Fig. 2. We see the eccentricity of Enceladus approach the predicted value.
For Enceladus and Dione in the current $e_{E}$-type 2:1 resonance, the equilibrium eccentricity is $e^{2} \approx(30.69 D)^{-1}$. The value of $D$ depends upon the unknown $k_{2}$ and $Q$ of the satellite. Conventionally, Kelvin's formula (Love, 1944),

$k_{2}=\frac{3 / 2}{1+\frac{19 \mu}{2 \rho g R}}$,

has been used to estimate Love numbers of small satellites, where $\mu$ is the rigidity, $\rho$ the density, $g$ the surface acceleration, and $R$ the radius. For Enceladus, taking $\mu=4 \times 10^{9} \mathrm{Nm}^{-2}$, we find $k_{2}=0.0018$. With an assumed $Q_{E}$ of Enceladus of 20, the equilibrium eccentricity of Enceladus is 0.014 . This is above the current eccentricity of 0.0047 ; so in this approximation, Enceladus is not in equilibrium and is still evolving deeper into resonance. Note that if the eccentricity is below the equilibrium eccentricity, and if the heat flow is steady, then the heating rate is lower than the equilibrium heating rate.

However, Ross and Schubert (1989) have shown that the dynamic Love number can be much larger than this conventional estimate. If the dynamic Love number is large enough then, in principle, Enceladus could be at a tidal equilibrium today. If Enceladus is at the equilibrium, then the estimates of the last section apply, and the heating in Enceladus is $1.1 \mathrm{GW}$ for a $Q_{S}=18,000$. If $Q_{S}$ is larger than this then the heating is proportionally smaller. Thus even with an enhanced dynamic $k_{2}$ the equilibrium heating rate is lower than the observed heat flux.

For which parameter values is the current Enceladus-Dione system at equilibrium? Given the current eccentricity of 0.0047 , and a value for $Q_{S}$, we can determine the required value of $k_{2 E} / Q_{E}$ for equilibrium. This is the solid curve shown in Fig. 3. Above this curve, the current eccentricity is above the equilibrium value and below this curve it is below the equilibrium value. The horizontal line shows the $k_{2 E} / Q_{E}$ for Kelvin's estimate of the Love number (calculated above) and for $Q_{E}=20$. We see that for this value the current system is at an equilibrium for $Q_{S}=159,000$. The equilibrium heating rate for the 2:1 Enceladus-Dione resonance as a function of $Q_{S}$ is also shown in Fig. 3.

Keep the "Mimas test" in mind. If $k_{2 E}$ is significantly enhanced over the Kelvin value because of the viscoelastic properties of ice, then one might expect this also to be the case for Mimas.

\section{Conclusion}

The rate of heating of Enceladus in an equilibrium resonant configuration with other saturnian satellites can be estimated independently of the physical properties of Enceladus. Our results update the values obtained for the equilibrium tidal heating found by Lissauer et al. (1984) and Peale (2003). We find that equilibrium tidal heating cannot account for the heat that is observed to be coming from Enceladus, and current heating rates are even less for conventional estimates of $k_{2 E}$. Even allowing a dynamic $k_{2 E}$ much larger than the conventional $k_{2 E}$, as can occur for viscoelastic models (Ross and Schubert, 1989), the equilibrium tidal heating is less than the heat observed to be coming from Enceladus.

One resolution is that the tidal equilibrium is unstable and that the system oscillates about equilibrium. Yoder (1981) suggested that Enceladus might oscillate about equilibrium if the $Q$ of Enceladus is stress dependent. An alternate suggestion was made by Ojakangas and Stevenson (1986), who emphasized the possible temperature dependence of $Q$. In these models Enceladus would now be releasing heat stored during a recent high eccentricity phase. There may be other mechanisms to produce episodic behavior. For instance, perhaps Enceladus could just store the tidal heat as the system evolves monotonically and release it episodically. These mechanisms may be consistent with the episodic character of the resurfacing events as suggested by spacecraft images. But it is curious that one has to appeal to nonequilibrium tidal oscillations or episodic activity to heat both Io and Enceladus (Ojakangas and Stevenson, 1986). If the fraction of time spent in an active state is, say, of order $20 \%$, for each satellite, then the probability that both are found in an active state today is only $4 \%$.

Other low-order resonance configurations are possible for the saturnian satellites in the past. These include the 3:2 Mimas-Enceladus and the 3:4 Enceladus-Tethys resonances. The latter resonance has no equilibrium because the orbits are diverging, and the former has an equilibrium heating of only $0.48 \mathrm{GW}$. So equilibrium heating at past resonances is no more successful at 
explaining past resurfacing events than equilibrium heating is at explaining the present activity.

\section{Acknowledgments}

We thank Oded Aharonson, Tony Dobrovolskis, Jack Lissauer, Re'em Sari, and Stan Peale for helpful conversations.

\section{References}

Gavrilov, S.V., Zharkov, V.N., 1977. Love numbers of the giant planets. Icarus 32, 443-449.

Kieffer, S.W., Lu, X., Bethke, C.M., Spencer, J.R., Marshak, S., Navrotsky, A., 2006. A clathrate reservoir hypothesis for Enceladus' south polar plume. Science 314, 1764-1766.

Lissauer, J., Peale, S.J., Cuzzi, J., 1984. Ring torque on Janus and the melting of Enceladus. Icarus 58, 159-168.

Love, A.E.H., 1944. A Treatise on the Mathematical Theory of Elasticity, fourth ed. Dover, New York.

Ojakangas, G.W., Stevenson, D.J., 1986. Episodic volcanism of tidally heated satellites with application to Io. Icarus 66, 341-358.

Peale, S.J., 2003. Tidally induced volcanism. Celest. Mech. Dynam. Astron. 87, 129-155.

Peale, S.J., Cassen, P., 1978. Contribution of tidal dissipation to lunar thermal history. Icarus 36, 245-269.
Porco, C.C., and 24 colleagues, 2006. Cassini observes the active south pole of Enceladus. Science 311, 1393-1401.

Ross, M.N., Schubert, G., 1989. Viscoelastic models of tidal heating in Enceladus. Icarus 78, 90-101.

Schubert, G., Spohn, T., Reynolds, R., 1986. Thermal histories, compositions and internal structures of the Moons of the Solar System. In: Burns, J., Matthews, M. (Eds.), Satellites. Univ. of Arizona, Tucson, pp. 224 292

Sinclair, A.T., 1983. A re-consideration of the evolution hypothesis of the origin of the resonances among Saturn's satellites. In: Markellos, V.V., Kozai, Y. (Eds.), Dynamical Trapping and Evolution in the Solar System. Reidel, Dordrecht, pp. 19-25.

Spencer, J.R., and 9 colleagues, 2006. Cassini encounters Enceladus: Background and the discovery of a south polar Hot Spot. Science 311, 14011405.

Squyres, S.W., Reynolds, R.T., Cassen, P.M., Peale, S.J., 1983. The evolution of Enceladus. Icarus 53, 319-331.

Stevenson, D.J., 2006. How does Enceladus do it? In: AGU Fall Meeting. Abstract P22B-03.

Wisdom, J., 2004. Spin-orbit secondary resonance dynamics of Enceladus. Astron. J. 128, 484-491.

Yoder, C.F., 1981. Tidal friction and Enceladus' anomalous surface. Eos 62, 939

Yoder, C.F., Synnott, S.P., Salo, H., 1989. Orbits and masses of Saturn's coorbiting satellites: Janus and Epimetheus. Astron. J. 98, 1875-1889. 